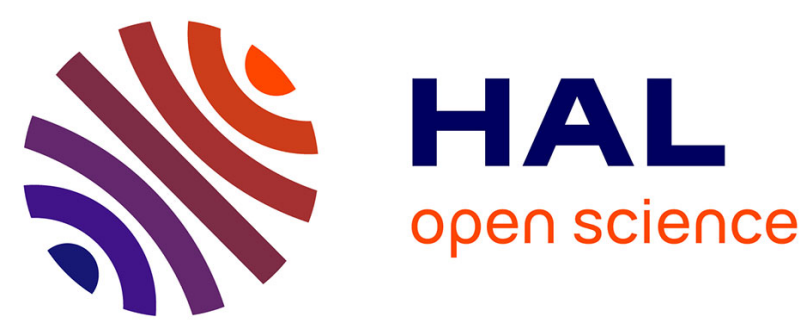

\title{
Jouer avec les mouvements, les vibrations et les rythmes dans l'émergence de la voix
}

\author{
Chantal Lheureux-Davidse
}

\section{To cite this version:}

Chantal Lheureux-Davidse. Jouer avec les mouvements, les vibrations et les rythmes dans l'émergence de la voix. Corps \& psychisme: recherches en psychanalyse et sciences , 2007, La voix, 4 (48), pp.185 - 203. 10.3917/cpsy.048.0185 . hal-01513842

\section{HAL Id: hal-01513842 \\ https://hal.science/hal-01513842}

Submitted on 25 Apr 2017

HAL is a multi-disciplinary open access archive for the deposit and dissemination of scientific research documents, whether they are published or not. The documents may come from teaching and research institutions in France or abroad, or from public or private research centers.
L'archive ouverte pluridisciplinaire HAL, est destinée au dépôt et à la diffusion de documents scientifiques de niveau recherche, publiés ou non, émanant des établissements d'enseignement et de recherche français ou étrangers, des laboratoires publics ou privés. 


\section{JOUER AVEC LES MOUVEMENTS, LES VIBRATIONS ET LES RYTHMES DANS L'ÉMERGENCE DE LA VOIX}

Chantal Lheureux-Davidse

L’Esprit du temps | « Champ psychosomatique »

2007/4 n 48 | pages 185 à 203

ISSN 1266-5371

ISBN 9782847951165

Article disponible en ligne à l'adresse :

http://www.cairn.info/revue-champ-psychosomatique-2007-4-page-185.htm

\section{Pour citer cet article :}

Chantal Lheureux-Davidse, « Jouer avec les mouvements, les vibrations et les rythmes dans l'émergence de la voix », Champ psychosomatique 2007/4 (n 48), p. 185-203.

DOI 10.3917/cpsy.048.0185

Distribution électronique Cairn.info pour L'Esprit du temps.

(C) L'Esprit du temps. Tous droits réservés pour tous pays.

La reproduction ou représentation de cet article, notamment par photocopie, n'est autorisée que dans les limites des conditions générales d'utilisation du site ou, le cas échéant, des conditions générales de la licence souscrite par votre établissement. Toute autre reproduction ou représentation, en tout ou partie, sous quelque forme et de quelque manière que ce soit, est interdite sauf accord préalable et écrit de l'éditeur, en dehors des cas prévus par la législation en vigueur en France. Il est précisé que son stockage dans une base de données est également interdit. 


\section{Jouer avec les mouvements, les vibrations et les rythmes dans l'émergence de la voix}

\author{
Chantal Lheureux-Davisdse
}

$\mathrm{L}$

orsque nous rencontrons un enfant autiste, en retrait dans son monde, nous nous demandons souvent s'il nous fera entendre le son de sa voix. Si l'enfant se sent exister en continu dans son corps, il se sent concerné par luimême et par les autres dans une communication partagée. Les recherches psychanalytiques sur l'autisme de Geneviève Haag1 nous sensibilisent à l'importance de l'investissement de l'image du corps dans la relation à l'autre, pour la constitution du moi corporel et au rôle des fixations précoces et des mouvements régressifs dans le développement relationnel, dans le cadre du transfert.

Donald Meltzer² a mis en évidence les mouvements d'investissement et de désinvestissement corporel qui vont de pair avec ce qu'il a nommé la réversibilité du démantèlement sensoriel. En effet, lorsque l'enfant autiste, submergé par un excès de sensations ou d'émotions, se clive de ses ressentis corporels pour tenter de s'apaiser, il quitte provisoirement le lien entre psyché et soma, pour se réfugier dans ses pensées. Ses pensées peuvent alors dériver peu à peu par résonance en perdant leurs liens logiques et contextuels. Ainsi la sensorialité de l'enfant se morcelle, et chaque sens devenant indépendant des autres, ne trouve plus de lien avec les autres sens. L'enfant ne peut plus écouter s'il regarde, et le tactile est parfois nécessaire pour accéder ensuite au visuel. Ce démantèlement sensoriel, bien qu'il soit réversible, est issu d'états de

Chantal Lheureux-Davidse, maître de Conférences Paris 7 Denis Diderot 59, Rue du Temple 75004 Paris
1. HAAG G., Contribution à la compréhension des identifications en jeu dans le moi corporel, Journal de psychanalyse de l'enfant, $\mathrm{n}^{\circ} 20 \mathrm{Le}$ corps, Bayard éditions, 1997, pp. 104-125

2. MELTZER D., 1975 , Explorations in Autism, Rolland Harris Trust, Clunie Press, trad. Franç., Explorations dans le monde de l'autisme, Paris : Payot, 1980, p. 259 
3. Les travaux de Suzanne Maiello sur le sonore prénatal sont très éclairants. La rythmicité des bruits cardiaques et de la respiration de la mère sont comme le support d'une expérience de continuité pour le foetus qui lui permet d'accéder ensuite

à l'imprévisibilité des bruits digestifs puis de la voix. MAIELLO S.,

L'objet sonore. Hypothèse d'une mémoire auditive prénatale, Journal de psychanalyse de l'enfant, $\mathrm{n}^{\circ} 20$, Le corps, Paris : Bayard éditions, 1997, pp. 40-66 saturation sensorielle qui font effraction et limite l'accès à la rencontre avec l'autre.

Lorsque ces installations en clivage durent trop longtemps, elles privent l'enfant du lien avec ses ressentis corporels et avec son sentiment d'exister. Son moi corporel s'efface et toute communication spontanée avec les autres devient problématique. L'enfant en perd le sens de l'altérité et parfois même la motricité physique devient difficile. Le démarrage d'un mouvement peut être alors impossible. Des vécus d'anéantissement peuvent surgir comme si le corps n'existait plus. L'image du corps se défait peu à peu dans des vécus d'arrachement des extrémités, des mains et des pieds, du bas du corps, de la bouche, et l'axe du dos ne maintient plus la tonicité du corps, le regard est ailleurs. Les expressions faciales sont comme gelées et toute communication par l'expression de sa voix semble devenir impossible.

Pour lutter contre des risques d'effondrement psychique ressentis corporellement comme un effondrement physique, l'enfant tente de s'auto-maintenir par une carapace musculaire qu'il met sous tension, qui fait office de seconde peau.

Les suivis psychothérapeutiques avec ces enfants rebutent de nombreux psychanalystes qui ne pensent pas toujours qu'une évolution serait envisageable dans ce cadre. Comment la rencontre serait-elle possible, particulièrement lorsque ceuxci ne manifestent aucun langage verbal et que le langage préverbal parait inexistant?

Mais faut-il attendre qu'un bébé émette un son avec sa voix pour avoir l'idée de lui parler? Lorsqu'un enfant autiste ne prononce pas un son, il n'est pas rare qu'il comprenne ce qu'on lui dit. Par contre, pour d'autres enfants sans langage verbal, la parole des autres n'est même pas supportable au point qu'ils se bouchent les oreilles.

L'imprévisibilité de la voix est parfois intolérable ${ }^{3}$. Les rythmes prévisibles des sons ou de la musique peuvent aussi être insupportables, particulièrement lorsque la musique est chantée.

Comment permettre à ces enfants d'accéder à une rencontre avec les autres alors qu'ils sont si profondément enfermés dans des états archaïques avant même que le sonore et le visuel soient intégrables? 


\section{VIBRATIONS ET SENTIMENT D’EXISTENCE}

C'est un groupe de quatre jeunes autistes entre quinze et vingt ans, sans langage, profondément en retrait, qui m'a offert quelques pistes de réflexions.

J'avais l'habitude de voir ces jeunes dans le cadre de leur groupe, hors de toute communication la plupart du temps, hypotoniques, ne réagissant que très peu à la voix. C'était un jour d'automne, deux horticulteurs étaient venus tailler les branches des arbustes à la tronçonneuse. Ce qui faisait un bruit infernal. C'est à ce moment-là que les quatre jeunes se sont levés pour se diriger d'eux-mêmes vers la courette, attirés par les vibrations des machines qui semblaient les réveiller. Côte à côte, leur corps étaient plus toniques, leurs visages s'éclairaient et devenaient expressifs, des regards et des sourires s'échangeaient spontanément dans une jubilation partagée. Mais dès que les bruits des machines marquaient un arrêt, les jeunes s'éteignaient et semblaient se flétrir, perdant tout tonus, pour se renfermer à nouveau. Élise était très enthousiaste ce jour-là et devenait joyeuse, alors qu'elle pleure beaucoup sans émettre le moindre son avec des troubles ORL chroniques d'écoulement nasal depuis sa naissance. Une rencontre deviendrait-elle possible pour eux par le biais de cette expérience sensorielle partagée des vibrations ${ }^{4}$ ?

Avec des enfants autistes sans langage verbal, il n'est pas rare que ceux-ci réagissent davantage aux vibrations des sons graves de la voix qu'aux sons aigus. Les sons graves font davantage vibrer le bas du corps alors que les aigus font vibrer le haut de la tête. Il devient pertinent d'appréhender le plaisir du langage verbal partagé par l'effet vibratoire des sons dans le corps, en deçà même de la question du sonore. Lors des premiers babillages, le bébé apprécie de faire vibrer ses lèvres pour jouer avec des vibrations sonores qu'il provoque. De même le bébé au développement normal joue avec des sons de sa voix qui font vibrer ses cavités buccales et nasales avec des «mmm», ou des «a-reuh». Puis ses pleurs se transforment parfois en plaisir sensoriel de productions sonores laissant la place à des essais mélodiques sous formes de gazouillis ${ }^{5}$.

Le bébé dès sa vie fœtale est davantage en relation avec sa mère si celle-ci a une voix «habitée ». En effet, une mère qui est bien reliée avec elle-même aura un timbre de voix vibrant avec des ancrages dans les graves. Sa voix sera également
4. J'avais suivi un bébé qui était resté fixé à des expériences sensorielles des vibrations avant l'intégration du sonore alors qu'il avait vécu les cinq derniers mois de grossesse dans la même poche à côté d'un jumeau mort. Après sa naissance, seules les vibrations de son environnement le rassuraient ainsi que le mouvement. Il semblait lutter contre le sommeil pour rassurer sa mère sur le fait que lui était bien vivant mais s'était ainsi épuisé jusqu'à présenter un gros retard de développement. LHEUREUXDAVIDSEC.,

Émergences du langage verbalchez des enfants autistes, Perspectives Psychiatriques,

Autismes : nouveaux enjeux cliniques et thérapeutiques, Paris: EDK, juillet-septembre 2006, n³, vol 45, pp. 226-230

5. Les travaux sur l'observation des bébés avec leur mère selon Esther Bick sont précieux pour repérer les fixations précoces chez des enfants en grande difficulté relationnelle. MEG HAARIS WILLIAMS, 1987, Collected Papers of Martha Harris and Esther Bick, London : The Dorian Harris Education Trust, The Clunie Press, Old Ballechin, Strahtay, Pertshire, trad fr. Jeanne et Jacques Pourrinet, Les écrits de Martha Harris et d'Esther Bick, collection Tavistock Clinic, Larmor-Plage : Editions du Hublot, 1998 
6. Les enfants autistes qui se mettent à parler ont parfois dans un premier temps une voix très aiguë. mélodieuse. Alors qu'une mère dépressive désinvestie corporellement, aura une voix plus sourde au timbre peu vibrant, comme si sa voix parlait d'ailleurs, dans un registre plus perché dans les aigus ${ }^{6}$. L'expression populaire disant qu'une relation nous fait vibrer, serait à prendre dans son sens littéral.

\section{JEUX PARTAGES AVEC LES VIBRATIONS ET LES VARIATIONS DE LA VOIX}

Dans la clinique de l'autisme, l'intérêt pour l'alternance des sons aigus et des sons graves de la voix traduit souvent un investissement du bas du corps dans une tentative d'accrochage du bas avec le haut du corps, lorsqu'il y avait un clivage horizontal pendant lequel seul le haut du corps était investi.

Avec les enfants autistes, l'expérience partagée de jeu avec les vibrations et celle de jeu avec les sons graves de la voix en particulier peuvent être un préalable nécessaire pour certains d'entre eux, à la découverte du plaisir des échanges langagiers : jeux avec les vibrations, les sons, les bruits, puis avec des voyelles et des consonnes, avec le rythme des mots, graves et aigus, forts ou chuchotés.

Ces expériences sonores avec leur voix tracent des directions dans l'espace des rencontres, du dehors au dedans, puis du dedans au dehors, traces de communications sensoriellement éprouvées à l'origine de formes.

Ces vibrations et ces sons, utilisables dans des circonstances différentes avec de légères variations, donnent l'expérience du langage dans une forme certes archaïque mais poétique, tel un jeu avec des mots qui résonnent avec d'autres par des sons communs, ou des rythmes identiques qui traduisent un plaisir certain dans la prononciation ou la scansion. Variations avec des voyelles et des consonnes éprouvées dans des circonstances différentes comme des mots qui peuvent changer de signification en fonction des variations du contexte. Lorsque les enfants autistes se mettent à parler, ils n'ont généralement pas de problèmes de prononciation. L'expression verbale ne passe pas toujours par les expériences langagières des bébés. Des mots voire des phrases peuvent être soudainement prononcés directement sans l'expérience des babillages.

Le choix de mots prononcés par les enfants autistes est souvent relié à un seul contexte et n'est pas toujours transpo- 
sable ailleurs. Les jeux sur les variations et les vibrations peuvent alors ébranler cette rigidité et peut ainsi favoriser l'accès au langage à condition que ces jeux de variations portent sur des registres suffisamment archaïques pour que l'enfant ne soit pas pris dans la confusion ou dans une saturation sensorielle.

Les travaux de Laurent Mottron ${ }^{7}$ sur l'écoute des enfants autistes démontrent qu'ils ont tendance à se focaliser sur les détails au détriment d'un ajustement en va et vient entre les détails et le contexte d'ensemble. Chaque détail devient alors une catégorie. Ainsi les enfants autistes accèdent difficilement à la notion de concept à cause d'une hyper-catégorisation de chaque détail. Par exemple le concept chat ne peut être appréhendé en dehors des expériences vécues de chaque chat rencontré. Ce qui explique que chaque chat dans sa particularité devient un concept à lui seul. C'est ainsi que la transposition d'une expérience vécue à un autre contexte reste problématique ${ }^{8}$.

Le jeu avec les variations, les vibrations et les sons ne propose-t-il pas au contraire l'expérience de changements de points de vue et de contextes? Cela devrait favoriser l'accès au langage verbal. De plus, d'un point de vue développemental, l'expérience des vibrations que vit le foetus précède (à 3 mois et demi de grossesse) l'expérience du sonore. En effet, la formation des sens et de l'ouie en particulier se situe au $4 \mathrm{e}$ mois de grossesse.

Il me semble judicieux dans un cadre thérapeutique de rester attentif aux expériences vibratoires auxquelles l'enfant autiste réagit, afin d'en faire une occasion de rencontre partagée avec le thérapeute, s'il en nomme l'intérêt.

\section{DIALOGUE PAR LE MOUVEMENT}

Dès le début du $4 \mathrm{e}$ mois de grossesse, le fotus dialogue in utero avec sa mère par l'alternance de leurs mouvements. En effet lorsque la mère bouge, le fœetus s' arrête de bouger jusqu'à ce que celle-ci se pose et le temps que son rythme cardiaque s'apaise pour retrouver son tempo. Il répond alors par le mouvement à la mesure des mouvements proposés par la mère précédemment. Ce sont de véritables dialogues dans l'alternance de mouvements.
7. MOTTRON L.,

L'autisme : une autre intelligence. Diagnostic, cognition et support des personnes autistes sans déficience intellectuelle, Belgique : Pierre

Mardaga éditeur, 2004

8. C'est ce que Temple Grandin explique dans son dernier livre : «Tous les chiens que je connaissais étaient d'assez grande taille et c'est comme ça que je les identifiais. Et puis nos voisins ont acheté un teckel, et cela m'a plongée dans une grande perplexité. Comment cette petite chose pouvait-elle être un chien? J'ai longuement observé le teckel pour tenter de résoudre cette énigme. Et j'ai fini par remarquer qu'il avait le même type de nez que mon golden river. C'était donc ça: les chiens ont des nez de chien .» GRANDIN T., L'interprète des animaux (traduit de l'anglais), Paris, Odile Jacob, 2006 
Tant que les enfants autistes en thérapie sont encore peu accessibles à la voix, ils peuvent être cependant hautement réceptifs aux échanges relationnels avec le thérapeute par les mouvements et les déplacements dans l'espace, dès lors que celui-ci a la capacité de jouer avec ces registres archaïques avec plaisir. Ils préparent ainsi une ouverture sur un langage plus élaboré par la suite qui peut prendre une forme verbale dans le meilleur des cas.

Il est toujours étonnant de constater combien les mères s'émerveillent de chaque mot, de chaque expression ou de chaque son avec leur bébé, commentant chaque mouvement ou déplacement, dans un à priori que tout est sujet au langage. Mais nous perdons trop souvent cette capacité avec des personnes en grande difficulté relationnelle comme avec les personnes autistes, déficientes ou handicapées.

\section{LE SONORE EFFRACTANT}

La rencontre thérapeutique avec Dorian est devenue possible à travers ces registres archaïques. Dorian est un garçon d'une dizaine d'année, il est handicapé moteur en fauteuil, dans une coquille moulée qui enveloppe tout son corps de la tête aux pieds. Seuls ses bras et sa tête restent mobiles. Son handicap moteur s'accompagne de troubles autistiques profonds sans langage verbal. Il ne supporte aucun bruit ni aucune musique. La vie en groupe le submerge. Les états de saturation sensorielle l'empêchent de filtrer ce qui lui parvient au point qu'il semble se dissoudre et se perdre dans son environnement qu'il vit comme effractant. Son raidissement musculaire en carapace tente de lutter contre des sensations d'effondrement corporel, mais provoque de telles tensions jusqu'aux crampes, qu'il en devient douloureux. Des tentatives d'implant d'une pompe à l'intérieur du ventre diffusant dans le canal rachidien un myorelaxant ont échoué à deux reprises, probablement par un rejet de son organisme qui ne peut pas accepter d'être relaxé sans craindre l'effondrement. Il tente de se décharger par des cris stridents et il vérifie sa solidité s'il se relaxe un instant en se tapant la mâchoire avec son poing ou en se mordant violemment la main. La rencontre individuelle dans un cadre psychothérapeutique lui est plus facile, car elle limite l'imprévisibilité de son environnement. L'enjeu était 
qu'il puisse arriver à se détendre sans risquer de s'effondrer, au bénéfice d'une relation qui s'installe, afin qu'une bonne différenciation entre dedans et dehors et qu'un pare-excitant se construisent. Je nommais depuis quelques temps tout ce qui pouvait être attrayant comme j'aurais pu le faire avec un bébé et Dorian semblait en apprécier les bienfaits. Je profitais des trajets d'un bâtiment à l'autre pendant que je poussais son fauteuil jusqu'à mon bureau, pour nommer les plaisirs sensoriels et esthétiques qui se présentaient, tout en soulignant en chantant parfois que c'était l'occasion de se sentir bien tranquille et bien relaxé. Dorian commençait alors à pousser de profonds soupirs auxquels je répondais en alternance. Le mot «tranquille » sera un mot qu'il reprendra de lui-même spontanément quelques mois plus tard, dans le cadre des séances. Il commençait à apprécier de se détendre en baillant abondamment dans des souffles profonds. Mais la détente musculaire l'inquiétait aussitôt au point de se taper violemment la mâchoire ou de se mordre la main. Je verbalisais l'inquiétude qu'il pouvait avoir de s'effondrer s'il était relaxé et la nécessité pour lui de vérifier s'il était solide en éprouvant la solidité de sa mâchoire, de ses os et de ses dents. Je lui proposais à portée de main une série d'animaux en caoutchouc souple mais ferme, qui lui permettait par imitation de mes gestes d'en éprouver la consistance élastique, douce et solide en même temps. Il semblait peu à peu s'approprier ces mêmes qualités pour lui-même et il commençait à profiter d'une détente tout en se sentant solide, sans plus se taper.

\section{BRUITS DU DEHORS ET BRUITS DU DEDANS}

$\mathrm{Au}$ retour dans son groupe, il retrouvait ses angoisses d'effraction sonore et se mettait à crier ou à se taper immédiatement. C'était le cas des jours de tempête lorsque la pluie frappait fort autant sur les vitres que dans ses tympans. Ce jour-là, je craignais qu'il ait recours à ces stratégies de décharge alors que nous entendions vrombir les machines pour souffler les feuilles dans le jardin. Je lui demandais alors si lui aussi pouvait faire ses bruits à lui qui parleraient des bruits gênants du dehors. Il me regarda en face et s'appliqua à produire volontairement un son avec sa voix : «brrrreeee ». Je le félicitai et je théatralisai que ses bruits du dedans étaient une 
façon de dire aux bruits de dehors qu'ils étaient gênants, afin qu'il repousse ces bruits très loin de lui. Il semblait très rassuré par cette proposition tout en en faisant l'expérience sans crier ni se taper. J'appris quelques jours plus tard que «Dorian n'allait pas bien du tout en ce moment». Je m'en étonnais puisque je ressentais exactement le contraire. Son éducatrice me précisa que depuis quelques jours, il faisait des bruits continus de vibrations graves pendant qu'il déjeunait. Je me réjouissais qu'il ait pu investir de lui-même cette technique dans un autre lieu aussi bruyant et effractant qu'est le réfectoire des enfants. Son éducatrice était alors rassurée en comprenant que Dorian commençait à se protéger des bruits extérieurs tout en accédant au langage. Il retrouvait également un accrochage solide dans sa verticalité en joignant ses mains spontanément et en alternant des mouvements de tête de gauche à droite que j'accompagnais d'une comptine improvisée en rythme sur le thème de la gauche qui rencontre la droite. Un accrochage des hémicorps autour d'une verticalité retrouvée lui donnait plus de tonicité sans qu'il s'auto-agrippe en carapace comme il le faisait autrement. Son regard s'installant peu à peu, il pouvait poser avec plus de confiance sa tête en arrière sur le support de son fauteuil normalement réservé à cet effet.

\section{EXPLORATION DE L'ESPACE PAR LA LANGUE, LES DENTS ET LE SOUFFLE}

Le réinvestissement de sa verticalité d'arrière-plan puis d'avant plan lui donna accès sur cet axe à sa bouche qui se tonifiait. Il était maintenant capable de fermer ses lèvres pour contenir sa salive et pour explorer quelques sensations dans sa cavité buccale. Je nommais le plaisir de la langue de se sentir en sécurité au milieu des dents bien solides au moment où des mouvements de langue incontrôlés tentaient de chercher une paroi contenante pour se situer dans l'espace.

La langue est le premier organe d'exploration de l'espace avant même que les membres puissent être opérants. Les travaux d'embryologie mettent en évidence en effet que la langue se forme en même temps que les quatre membres, à partir de la même zone qui migre du tronc cérébral. C'est ainsi que la langue et les membres ont par leurs origines communes 
de temps et de lieu de leur formation, des fonctions communes d'exploration de l'espace.

Dorian se mit à explorer l'espace buccal avec sa langue, comme s'il intériorisait une sécurité retrouvée. Ce qui lui permit de prononcer des «ah » et des «oh» qu'il accompagnait de son souffle comme pour expérimenter des directions du son de l'intérieur à l'extérieur de sa bouche. Je commentais tous ces plaisirs de sons associés à sa respiration. Entendant le mot «respiration», Dorian attrapa un petit cochon en caoutchouc qu'il porta à son nez pour le respirer et se mit à dire «qu'est-ce que ça sent?», puis «le cochon». Puis il éprouva les vibrations dues aux chocs qu'il provoqua en claquant ses mâchoires entre elles. Mon esprit tint alors compte de la littéralité de ce que j'énonçai lorsque je lui parlais des bruits de «dedans » comme s'il s'agissait de bruit de «deux-dents ». Les bruits tout doux de la respiration qui sortaient de la bouche pouvaient exister dans la rencontre des sons de dents bien solides9. Métaphoriquement je me représentais qu'il pouvait exister au milieu d'une cavité que représentait la bouche, dans une naissance enfin douce comme un souffle, issu de bons fantasmes de rencontre primitive qu'il éprouvait par le claquement des mâchoires. Dorian était né prématurément et avait subi plus d'un mois de ventilation en réanimation. Son handicap était dû à des séquelles de prématurité.

Dorian expérimente ensuite des sons doux comme «ya!» et des sons plus durs qui se prononcent avec les dents comme « tata, mata, ta» en les accompagnant de bruits de claquement de dents.

\section{AGRESSIVITÉ ORALE ET FANTASME DE SCÈNE PRIMITIVE}

Je pense alors à la difficulté que Dorian peut avoir à croquer et à mastiquer les aliments durs en lien avec sa difficulté à investir l'agressivité. Son alimentation est essentiellement faite de nourriture sucrée, liquide ou moulinée qu'il a tendance à téter. Ce que l'on retrouve souvent chez certains enfants fixés à une oralité pré-ambivalente, avant l'apparition des dents. Une séance suivante, je dispose deux balles en caoutchouc souple avec des aspérités qu'il écrase entre ses mains, qui me font penser à des mandibules qui écrasent avec
9. Les travaux de recherche en orthodontie de Bruno BONNET sont très éclairants, concernant la difficulté de certains enfants à investir la mastication et la fonction de la langue dans la cavité buccale. 
plaisir les aliments, en disant «deux balles », puis articule deux autres mots : mon prénom «Chantal» et celui de «Johanna», la stagiaire psychologue qui assiste aux séances. Je faisais remarquer à Dorian que tous ces mots « deux balles, Chantal, Johanna » sont drôlement amusants à prononcer parce qu'au milieu de chacun de ces mots il y a le son «a» qui a l'air de se sentir bien confortable au milieu. Il jubile et les répète spontanément avec plaisir. Le «a» au milieu des mots semble faire résonance avec les vibrations éprouvées au milieu du corps. Il investit alors un œuf à rythme qu'il place dans son axe en disant «œuf», qu'il fait passer en alternance d'une main à l'autre, à la manière des bébés qui font passer un hochet avec beaucoup de concentration d'une main à l'autre, en éprouvant les vibrations sonores de l'intérieur du jouet. Ces mouvements d'alternance de gauche à droite participent à la construction de la charnière verticale de leur corps qui accroche les hémicorps gauche et droite.

À la séance suivante, Dorian répète parfaitement avec plaisir pendant tout le trajet «Johanna» en mémoire des jeux de sons que nous avions faits à la séance précédente. Il semble éprouver avec plaisir la scansion en trois syllabes avec le «a au centre du mot. J'ai l'impression d'occuper dans le transfert une des extrémités du mot et que Johanna occupe l'autre extrémité pour créer la place au centre pour Dorian représenté par le «a du milieu. Dorian joue alors avec ces trois syllabes en disant: «Jo-ha-nna, Jo-nna, ha-nna, Jo-ha-nna», dans une conscience poétique de la place de chaque son, en les faisant varier pour former d'autres associations de syllabes. J'ai l'impression que le «a» du milieu le représentait par résonance au milieu de ses parents et qu'il éprouvait par ces jeux sa place et son existence issue de la rencontre de ses parents comme lorsqu'il disait «Jo-nna» en ayant pris soin de ne pas mettre le «a» du milieu. Ce jour-là nous avons croisé pendant le trajet quelques personnes de l'institution, et nous avons joué à prononcer les prénoms à trois syllabes: «Jo-hanna, Jo-na-than ». Mais il n'a jamais dit certains prénoms qui ne comprenaient pas les mêmes voyelles.

En séance, Dorian s'est ensuite amusé à faire vibrer des sons graves dans la gorge, en les alternant avec des claquements de dents, des sons doux voyelliques et des sons consonnantiques durs.

Sur le trajet du retour, nous rencontrons en chemin sa 
kinésithérapeute Anne-Marie et mon enthousiasme m'a autorisée à partager avec elle le bonheur que nous avions eu à prononcer les prénoms des personnes avec Dorian. Celle-ci me disait que Dorian n'avait jamais pu prononcer plus que la moitié de son prénom «Marie», ce à quoi Dorian répondit «Anne-Marie». Comme si désormais les deux moitiés pouvaient exister simultanément dans la rencontre et la différenciation ${ }^{10}$.

\section{JEUX DE SONS ET JEUX DE MOTS PARTAGÉS}

Dorian attendit autant que moi la séance suivante l'occasion de jouer avec les sons des mots. Nous assistons alors à un véritable feu d'artifice de mots, qu'il lance dans une liberté associative au cours de nos échanges, qui nous a laissés tout trois très émus.

Dès le trajet, Dorian prononce avec un plaisir certain « Joha-nna», comme s'il était surpris lui aussi d'entendre sa voix. En entrant dans le bureau, il aperçoit un crocodile articulé dont j'actionne la bouche et qui se met à couïner. Je dis que le crocodile aimerait bien parler mais qu'il ne sait faire que des bruits amusants avec sa bouche. Dorian essaie aussitôt de faire lui aussi des bruits avec sa bouche tout en fermant les yeux pour mieux en ressentir les vibrations et la spatialisation des sons : «a-u-iu, en». Il est ensuite distrait par des bruits d'une salle de bain voisine et par des bruits d'enfants qui passent dans le couloir. Je lui demande si lui aussi a des bruits de dedans qui parleraient des bruits entendus dehors. Il répond distinctement: «salle de bain» puis il alterne des sons qu'il fait vibrer pour le plaisir dans la bouche et des mots articulés qu'il semble choisir avec précision: «il y a- long-temps, Jona-than, un é-véne-ment», puis «aï-keuill-keuill».

En écoutant et en répétant ces associations de mots, je remarque la prononciation scandée en trois temps avec des sons communs «a»et «en». Je le lui fais remarquer et je reprends les mots qu'il avait déjà prononcés en les chantant et en les scandant. Il continue son improvisation en disant: « ouan, o-or», puis «dada». J'ai l'impression d'un bébé pris dans le plaisir du babillage, ce que je nomme en disant qu'il est bien agréable de jouer avec les bruits dans la bouche pour le plaisir. Il sourit en me regardant et déplace légèrement son fauteuil
10. HAAG G., La mère et le bébé dans les deux moitiés du corps, Neuropsychiatrie de l'Enfance, 1985, 33, pp. 107-114 
vers la gauche en disant «à gauche, la voiture, tranquille, dada ». Puis il s'exerce à des «ah » et des «oh» qu'il fait sortir de sa bouche sur un souffle comme il avait pu le faire à d'autres reprises. Je lui dis qu'il a toujours des réserves de bruits et de mots à l'intérieur, que l'on peut en fabriquer toute sa vie et que cela fait une bonne réserve. Il semble soulagé de n'avoir pas à se vider en se mettant à parler et baille, marquant ainsi une détente profonde.

Il essaie ensuite volontairement la prononciation d'une nouvelle lettre : « $\mathrm{r} »$. Son langage semble alors davantage organisé. Je l'imite en commentant ce plaisir nouveau. Il enchaîne par d'autres mots : «la forêt, l'autoroute» qui contiennent également la lettre « $\mathrm{r} »$. Johanna ajoute «crocodile » au répertoire des mots en $« \mathrm{r} »$. Dorian poursuit en disant : «mot de passe, les mots » et «broum-broum». Nous cherchons les lettres en commun entre tous ces mots choisis et découvrons avec émerveillement que les lettres communes sont en fait exactement celles de son prénom et de son nom, comme s'il se préparait ainsi avec ces sons à pouvoir prononcer un jour son nom. Par la suite il me demanda de changer le son de la mélodie du piano tout en m'attrappant la main, dans une recherche de variations en disant «son» puis «le son » et «changer le son ». J'improvise alors des associations de «sons, chanson, changer le son, sur le pont d'Avignon...»

\section{VIBRATIONS ET INTÉRIORISATION}

Il jubile mais semble débordé d'émotions nouvelles avec tous ces mots. Il tente de se récupérer par des babillages «aeu, a-eu », et se mord la main. Il nomme ses débordements en disant «oh la la, ah la la !». Cela l'apaise. Il dit alors: «son, musique, changer le son, nouvelle musique ». Puis il se bouche les oreilles, ferme les yeux et s'exerce avec des sons très intériorisés qu'il fait vibrer pour lui-même : «elle, sons, o, rr, a aommm ». Il prononce ces sons du dedans avec jubilation, dans un moment d'intériorisation des vécus précédents, par le moyen de sons qu'il s'approprie.

Je reprends le «aommm» qui me fait penser à un son très intériorisé répété inlassablement dans les pratiques religieuses de l'Orient. Ce son intériorisé fait vibrer tout le corps à partir 
$\mathrm{du}$ « $\mathrm{a}$ » ouvert sur l'extérieur et du «o » fermé plus intériorisé, prononcés au centre de la bouche et prolongé par le «mmm» qui diffuse des vibrations dans les cavités buccales et nasales puis dans tout le corps. Je le lui dis et il me répond par un prénom comprenant les mêmes lettres que le «a-o-mmm»: «Marie-Odile», jeune fille autiste dont je m'occupe également depuis des années. Nous nous émerveillons de cette séance. Je lui dis combien c'est formidable d'échanger avec lui, ce à quoi il me répond par un geste du pouce qui signifie «c'est super». Je commente et imite son geste. Prenant ainsi conscience de son geste, il regarde sa main tout en refaisant son geste, et jubile. J'ai le sentiment qu'une consensualité entre le tactile et le visuel devient possible.

La rencontre entre les sens dans ce que Donald Meltzer a nommé la consensualité fait écho, me semble-t-il, à la mise en réseau des syllabes, des sons et des mots dans le langage, condition d'accès au plaisir de la communication partagée.

Si Dorian utilise encore un registre de mots peu étendu il parait en connaître bien davantage. Il entre cependant dans le langage verbal avec plaisir dans des jeux de variations de sons, de syllabes et de mots qu'il emploie dans des registres différents, comme dans des jeux de rencontres improvisées et spontanées.

Le passage par de tels échanges, mais dans un premier temps dans le registre très archaïque des vibrations, des sons graves et des alternances de mouvements, lui a permis de faire des expériences de jubilation partagée sans être submergé par une avalanche d'informations sensorielles trop complexes, tant que la consensualité n'était pas possible.

Les réponses aux bruits extérieurs pénibles par des «bruits du dedans » avec sa voix lui ont donné l'expérience de s'isoler dans un premier temps d'un risque d'effraction du sonore ${ }^{11}$ pour ensuite constituer un pare-excitation capable de filtrer ce qui ne lui convient pas, pour maintenir un équilibre et une rencontre possible entre un dedans et un dehors, autrement que par un clivage radical.

Son mode d'apaisement par décharge de cris, de coups qu'il s'affligeait et d'auto-mutilation de ses mains, s'est transformé peu à peu en apaisement plus doux, à partir du moment où un dialogue devenait possible entre les registres opposés doux/solide, dedans/dehors, gauche/droite, entre lui et son environnement, par un regard accompagné de sourires, et par
11. C'est souvent pour cette raison que des enfants autistes se rassurent, lorsque les bruits ou les voix leur sont insupportables, en grinçant des dents. Le grincement de dents peut aussi avoir la fonction d'une réassurance que le mouvement et le son existent face à des sentiments de risque d'anéantissement. 
un dialogue verbal qui s'est installé spontanément entre les mots et le babillage.

Le travail sur la reconstruction de l'image du corps a participé à ce que Dorian se sente plus concerné par lui-même et par les autres. Cela n'a été possible cependant dans un premier temps que dans un cadre thérapeutique individuel dans lequel l'imprévisibilité de son environnement a été réduite. Cela devient transposable progressivement dans le cadre de son groupe où il commence à utiliser sa voix de plus en plus souvent.

\section{DIFFÉRENCIATION ET AUTONOMIE PARTAGÉE}

Ses cris, ses coups, son auto-mutilation et sa dispersion psychique sont remplacés peu à peu par un investissement relationnel et du langage à la condition qu'il soit assuré d'une bonne différenciation entre chacun pour éviter tout risque d'effraction.

Lors d'une improvisation musicale dans le cadre des séances nous étions allés chercher des instruments dans le placard. J'avais associé chaque instrument à chacun en marquant la différence des générations entre lui et les deux adultes (la stagiaire et moi-même) en disant: " c'est le tambour de la grande dame Johanna, le grand piano de Chantal et les clochettes d'un jeune garçon ». Il avait réagi avec un grand soupir de soulagement et il nous avait regardé attentivement en souriant. Puis il a dit: «jeune garçon», comme s'il était rassuré de sa différence générationnelle dans le cadre transférentiel.

Dorian qui ne supporte encore aucune musique dans son groupe, en réclame pendant les séances. Il appuie de lui-même sur un bouton du piano qui déclenche une mélodie pré-enregistrée qui passe en continu. Lorsque je constate que cela le submerge je lui propose de réduire l'intensité ou de diminuer la vitesse jusqu'à ce que le tempo lui convienne. Il fait ainsi l'expérience d'un apaisement possible sans recourir à une décharge trop coûteuse psychiquement. J'interprète qu'il pourrait peut être faire la même chose quand il y a trop de bruit gênant. Il soupire, baille dans une bonne détente et appuie tranquillement sa tête sur son dossier en disant «tranquille».

Je mesurai à quel point Dorian se sentait envahi dans son 
groupe par son environnement, sans qu'il puisse filtrer ses perceptions visuelles ou sonores ni même les mouvements. Dans le cadre transférentiel, je me surprenais ce jour à l'envahir de jouets que je disposais devant lui jusqu'à ce qu'il en soit saturé. Il commençait à se désorganiser pour lutter contre cet envahissement, tant corporellement que psychiquement. Sa tête, ses mains et sa langue adoptaient alors des mouvements désordonnés qui tentaient de s'accrocher en adhésivité sur les parois comme pour éviter un risque d'effondrement. Il était sur le point de recourir à des cris de décharge accompagnés de coups sur la mâchoire qu'il s'apprêtait à se donner. Je nommais ma responsabilité dans cet envahissement et dans sa lutte effrénée pour y faire face. En ayant ainsi attiré le transfert négatif sur moi, Dorian s'apaisa aussitôt. Il m'adressa un sourire reconnaissant comme s'il était enfin déchargé d'une lourde responsabilité. Je lui disais qu'il se croyait souvent obligé de s'intéresser à tout ce que je lui proposais tant au niveau visuel que sonore, sans doute pour me protéger et pour ne pas me laisser tomber, et que tout cela semblait l'épuiser et lui faisait oublier qu'il pouvait filtrer ce qui lui convenait. Son regard se plongeait avec bonheur dans les nôtres et il commençait à jouer avec plaisir de son côté comme un petit enfant. Il ne manquait pas alors de chercher à nous imiter ${ }^{12}$. Et nous prolongions le jeu en l'imitant également à distance, pour ensuite nous rencontrer par le biais des jouets dans un va et vient entre des retrouvailles et une certaine indépendance. Il pouvait désormais choisir ce qui lui plaisait et oublier le reste sans que cela me mette en danger. Nous nous exercions tous trois à choisir un jouet qui nous intéressait pendant que je nommais que nous n'avions pas à nous préoccuper des autres jouets. Dorian jubilait de ne plus avoir à s'inquiéter de moi comme il avait pu le faire auparavant. Je le rassurais en m'engageant à m'occuper de moi-même, seule ou avec l'aide de la stagiaire Johanna avec qui je formais symboliquement un couple parental dans le cadre transférentiel. Il faisait l'expérience de profiter du jouet qu'il avait choisi. J'entendais le son de sa voix de plus en plus souvent de façon appropriée. Nous jouions tous trois, seuls en présence les uns des autres, capables de filtrer les perceptions venant de l'environnement en fonction de l'intérêt de chacun, dans une autonomie partagée. Il appréciait particulièrement les échanges entre adultes que nous théâtralisions par des rencontres de
12 Les travaux de Jacqueline Nadel au CNRS sur l'imitation chez les enfants autistes sont très éclairants, notamment dans l'alternance imiter/être imité et dans l'importance de la spontanéité de l'imitation sans qu'elle soit demandée. NADEL J., DECETY J. Imiter pour découvrir l'humain, Paris : PUF, 2002 
jouets et par le langage. Cela semblait ainsi renforcer sa concevabilité et son sentiment d'existence.

Les moments intermédiaires et de changement ont été particulièrement investis non plus comme des moments de coupure catastrophique mais comme des occasions de liens par le langage, ce qui lui a permis de se sentir exister dans une certaine continuité.

Les rencontres entre l'équipe éducative de son groupe et moi-même, ainsi que les échanges dans le cadre des séances avec la stagiaire qui assistait à la thérapie ont favorisé dans le transfert la fantasmatisation pour Dorian d'une bonne scène primitive, à l'issue de laquelle il peut davantage se sentir exister et trouver sa place dans l'espace, issu d'un bon lien entre ses parents.

\section{DÉPRESSION PRIMAIRE ET ÉMERGENCE DU LANGAGE}

Ainsi l'équipe éducative a pu accompagner au mieux Dorian pendant cette émergence du langage qui a remis en scène une dépression primaire de sa vie de bébé qui n'avait pas encore été élaborée. Dorian a en effet passé quelques semaines à pleurer dans son groupe.

J'étais passée un jour dans son groupe alors qu'il pleurait de la sorte. Il était sur le sol hors de la coquille de son fauteuil, sur les genoux, pleurant de ne pouvoir se redresser comme il le souhaitait. Il pleurait comme un bébé en détresse n'ayant pas l'idée d'appeler ni de se faire consoler. Il ne pensait pas appeler et n'avait comme seuls interlocuteurs que lui-même et sa détresse. Je m'approchais de lui et nommais qu'il se sentait tout perdu, qu'il cherchait à se redresser sans y arriver en raison de son handicap moteur, perdant l'équilibre alors qu'il avait envie de se sentir bien grand. Je lui proposais de s'appuyer sur moi pour qu'il puisse mettre son buste à la verticale. Et je lui disais sous le regard attentif de son éducatrice que lorsqu'il pleurait ce n'était pas toujours facile de savoir ce qui n'allait pas et que son éducatrice comprendrait peut-être mieux s'il l'appelait en parlant. Il continuait à pleurer voulant être plutôt reconnu dans ses pleurs de bébé en détresse. Puis je lui proposais d'appeler son éducatrice en chuchotant: «Bénédicte!». Dorian s'arrêta de pleurer et prononça en 
chuchotant le nom de son éducatrice plusieurs fois. Elle se manifesta aussitôt en le félicitant de l'avoir ainsi appelée. La traversée d'une dépression primaire relance en effet, lorsqu'elle est bien accompagnée, la possibilité d'une émergence de la voix et du langage verbal, des liens et de la communication.

Le travail de nomination de la sensorialité vécue dans sa bouche lui a donné l'occasion d'expériences archaïques de la zone orale primitive, et a relancé l'investissement d'une oralité cannibalique dans le plaisir d'une agressivité avec ses dents. C'est ainsi qu'une certaine ambivalence a pu commencer à se construire, afin que les opposés soient non plus clivés mais en dialogue dans une bonne intégrité de son moi corporel. L'investissemnt de la bouche est une des conditions pour que l'enfant puisse se sentir concerné pour parler.

À la lumière du suivi de Dorian, il me paraît essentiel de constater que les enfants autistes qui ne supportent pas encore la voix des autres, ni même le sonore rythmique que l'on retrouve dans la musique, fixés à des expériences antérieures, comprennent cependant le langage verbal dès lors que le thérapeute leur parle de ce qui les intéresse. Leurs expériences concernent parfois des registres très archaïques comme celui des vibrations ou des mouvements. Un partage émotionnel devient ainsi possible et restaure un moi corporel effacé ${ }^{13}$. La bouche devient le terrain d'exploration de l'espace, de sensations et de vibrations, qui favorise l'advenue du langage dans la rencontre avec l'autre.

Bien que la formation de l'intégration sensorielle suive au début de l'existence des étapes chronologiques dans son développement, chacune de ces étapes peut être réinterrogée à toute occasion par la suite, non plus d'un point de vue développemental, mais de façon parallèle.

Nous pouvons également rester attentifs au cours de l'analyse de patients névrosés avec des fixations précoces, à ces éléments sensoriels ainsi qu' aux composantes vibratoires de la voix.
13. Les travaux de Colwyn Trevarthen sur le rôle des émotions partagées très tôt nous fournissent de nombreuses pistes sur les conditions d'accès au langage verbal. TREVARTHEN C., «Racines du langage avant la parole», in Devenir, vol. $9, \mathrm{n}^{\circ} 3$, pp. 73-93 


\section{BIBLIOGRAPHIE}

GRANDIN T., L'interprète des animaux, (traduit de l'anglais), Paris : Odile Jacob, 2006

HAAG G., «La mère et le bébé dans les deux moitiés du corps », Neuropsychiatrie de l'Enfance, 1985, 33, pp. 107-114

HAAG G., «Contribution à la compréhension des identifications en jeu dans le moi corporel », Journal de psychanalyse de l'enfant, n' 20 Le corps, Bayard éditions, 1997, pp. 104-125

MEG HAARIS WILLIAMS, 1987, Collected Papers of Martha Harris and Esther Bick, London: The Dorian Harris Education Trust, The Clunie Press, Old Ballechin, Strahtay, Pertshire, trad fr. Jeanne et Jacques Pourrinet, Les écrits de Martha Harris et d'Esther Bick, collection Tavistock Clinic, Larmor-Plage : Éditions du Hublot, 1998

LHEUREUX-DAVIDSE C., "Émergences du langage verbal chez des enfants autistes », Perspectives Psychiatriques, Autismes : nouveaux enjeux cliniques et thérapeutiques, Paris : EDK, juillet-septembre 2006, $\mathrm{n}^{\circ} 3$, vol 45, pp. 226-230

LHEUREUX-DAVIDSE C., L'autisme infantile ou le bruit de la rencontre, éditions Paris : L'Harmattan, 2003, 342 p.

MAIELLO S., «L'objet sonore. Hypothèse d'une mémoire auditive prénatale », journal de psychanalyse de l'enfant, $\mathrm{n}^{\circ} 20$, Le corps, Paris : Bayard éditions, 1997, pp. 40-66

MELTZER D., 1975, Explorations in Autism, Rolland Harris Trust, Clunie Press, trad. Franç., Explorations dans le monde de l'autisme, Paris : Payot, 1980, $259 \mathrm{p}$

MOTTRON L., L'autisme: une autre intelligence. Diagnostic, cognition et support des personnes autistes sans déficience intellectuelle, Belgique: Pierre Mardaga éditeur, 2004

NADEL J., DECETY J. Imiter pour découvrir l'humain, Paris : PUF; 2002

TREVARTHEN C., «Racines du langage avant la parole», in Devenir, vol. 9, $\mathrm{n}^{\circ} 3$, pp. 73-93

\section{RÉSUMÉ}

À partir de la clinique avec des enfants et des adolescents sans langage verbal avec des composantes autistiques, le rôle des échanges dans l'alternance de mouvements, les vibrations, la variation des rythmes sonores ou gestuels et les bruitages prennent une part certaine dans l'émergence de la communication.

Le plaisir de jouer dans ces registres archaïques participe à une réassurance dans le lien à l'autre lorsque le thérapeute en saisit le sens dans le transfert. En effet, en retrouvant des expériences prénatales d'alternance du mouvement et d'immobilité entre le fotus et la mère, dès le quatrième mois de grossesse, l'enfant en rupture de communication peut contacter une nouvelle opportunité d'échanges sans que tous les sens soient sollicités. De même, l'intérêt que ces enfants portent aux vibrations puis aux rythmes gestuels ou sonores, donne des perspectives de rencontres dans l'échange, 
avant même que la voix humaine puisse être intégrée. Les jeux sur la vitesse et l'intensité des mouvements, vibrations, bruits et rythmes permettent de mesurer avec finesse leurs seuils de tolérance et font écho aux expériences sonores et tactiles in utero, avant les moments catastrophiques.

La nomination dans la relation transférentielle, des impressions, des sensations et des émotions que l'enfant manifeste, favorise l'accès au langage verbal et aux échanges visuels. Ces premiers jeux dans le partage émotionnel que propose le thérapeute, à partir de ce que le patient donne à voir ou à entendre, participent à l'émergence du sentiment d'exister corporellement dans la continuité à l'origine de la capacité de communication.

Mots-clés : jeu - autisme - mouvements - vibrations - bruits - rythmes - voix - langage

\section{SUMMARY}

Based on clinical experience with children and adolescents without verbal language and autistic traits, discusses the role of alternating movements, vibrations, variations in rhythms of sounds or gestures in the emergence of language.

The pleasure to play in certain archaic registers contributes to a reassurance of the relationship with the other when the therapist grasps the meaning in the transfer. By contacting the pre-natal experience of alternation of movement and immobility between the fœtus and the mother, as of the $4^{\text {th }}$ month of pregnancy, the child with a rupture in communication may find a new opportunity to exchange, without all senses being involved. Similary these children may be interested in vibrations, then rhythms of gestures or sounds, which gives a perspective to meet in the exchange, before the human voice can be integrated. Playing with the speed, the intensity of the movements, vibrations, sounds and rhythms allows to measure precisely the tolerance level, and echo intra utero acoustic and tactile experiences, before the catastrophic moments.

Naming of impressions, sensations and emotions that the child manifests in the transfer relation helps him to access verbal language and visual interaction. The first experiences with playing ans sharing emotionally that is offered by the therapist, starting from what the patient shows or the sounds he makes, contribute to the emergence of felling to exist continuously in the body which is forms the origin of the capacity to communicate.

Key-words : play - autism - movement - vibration - sound - rhythm Voice - language 